\title{
Evaluación de algoritmos para estimar la derivada de presión en pruebas de pozos $^{*}$
}

Rubén Alberto González López**

\section{Resumen}

Aunque hay muchos métodos para hallar la función derivada que brindan buenos resultados en otros escenarios, en ocasiones la naturaleza discreta de las curvas de presión de pozo hacen que el procesamiento de éstas no sea adecuado. Además el ruido que le imprimen a la prueba las herramientas con la que se adquieren los datos y otros fenómenos naturales ajenos al yacimiento, dificulta el análisis y la posterior interpretación de la prueba.

Para determinar la función derivada de presión, es bastante común encontrar en la literatura los métodos de Bourdet, Simons y Horne. Últimamente se han utilizado con gran éxito algoritmos polinomiales como el de Spline y otros, no sólo, para hallar la función derivada de presión si no también, para filtrar los datos de la prueba de presión y así poder disminuirlas sobre presiones anormales que muchas veces hacen que éstas no se puedan interpretar.

Laexploración de los métodos polinomiales han permitido evaluarlos cualitativay cuantitativamente para compararlos con casos teóricos y de campo. Los métodos de mínimos cuadrados móviles y Savitzky - Golay se aplican tanto como filtro y como método derivativo. Ași mișmo, el método de la familia de los Spline, el Spline Periódico. En todos ellos el arreglo de puntos P us t se divide en tramos más pequerios ya que ninguna curva de presión se ajusta a una función polinómica.

El método de Savitzky - Golay, es muy útil como filtro para reducir el ruido de las pruebas, aunque utilizándolo para hallar la derivada, presentó inconsistencias en la parte del almacenamiento de la prueba, pero para tiempos tardíos resultó ser bastante apropiado. El método de mínimos cuadrados móviles, es recomendable para hallar la función derivada de presión, aunque basta que el polinomio de aproximación sea de grado 3 mientras que la ventana de los datos tratados se recomienda que sea grande. Por último, el método de Spline Periódico no se recomienda para determinar la derivada de pruebas de presión, pues los datos que se obtienen no muestran una tendencia satisfactoria.

\footnotetext{
* Fredty Humberto Escobar. Dinector proyecto de grado, M. Ph.D. Ingenienía de Ptróleos. Vicernector de huestigación y Proyección Social. Universidad Surcolombiana. fescobav@usco. edu.co * Ingeniaro de Petróleos. Universidad Surcolombiana. 
Palabras Clave: Pruebas de Presión, Función Derivada de Presión, Filtro Digital, Ruido, Mínimos Cuadrados Móviles, Aproximación Polinómica, Sistema de Ecuaciones, Funciones Polinómicas, Spline Periódico.

\section{Abstract}

Even though, there exist several methods for estimating the pressure derivative function which work well kin other scenarios, sometimes, the discrete nature of the well pressure curves makes its application somehow inadequate. Moreover the noise caused by the tools and other external naturalphenomena on the recorded data make the analysis and the subsequent test's interpretation to be difficult tasks.

It is very common to find different methods for estimation of the pressure derivative function. Such techniques as Bourdet, Simons and Horne, and recently the polynomial algorithms, v.g. Spline and others have been used successfully, not only to calculate the pressure derivative function but also to filter the pressure test data so that the abnormal pressures that many times makes the test non interpretable could be reduced.

Exploration of the polynomial methods has permitted their qualitative and quantitative evaluation so they could be compared with both theoretical and field cases. The Moving Least Squares and Savitzky - Golay methods are applied as both filter and derivative methods. Same situation takes place with method of the Spline family: the Periodic Spline. In all of the just mentioned methods the grouping of pressure versus time data points where divided into smaller sections since any pressure trend perfectly matches none of the known polynomial function.

The Savitzky - Golay method is very useful as a filter to reduce the noise introduced to the pressure tests. However, when determining the pressure derivative, it displays a high inconsistency at early time - wellbore storage - but, for later times in the test, it results to be very appropriate. The Moving Least Squares methods are recommended to find the pressure derivative function. It is enough to use a 3rd degree polynomial approximation while keeping wide the data window. Finally, the Peribdic Spline Method is not recommended to find the pressure derivative because the obtained data does not follow a satisfactory trend.

Keywords: Pressure Test, Pressure Derivative Function Digital Filter, Noise, Savitzky - Golay Moving Least Squares; polynomial approximation, Ecuations System, Polynomial Functions; Periodic Spline.

\section{Introducción}

【 na herramienta que ha llegado a engrosar el repertorio de utilidades de un Ingeniero de Yacimientos cuando interpreta pruebas de presión de pozo es la Tiab's Direct Sintesys Technique. De una manera practica ésta nos ayuda a calcular los parámetros para caracterizar los yacimiento de hidrocarburos.

Un factor importante para tener éxito en la interpretación de las pruebas de pozo con la Tiab's Direct Sintesys Technique, es el cálculo de la derivada de presión, y antes de obtener esta función se debe tener en cuenta que las pruebas están afectadas por distintos fenómenos que muchas veces hacen las pruebas muy dificiles de interpretar. Esto llevó a evaluar, distintos métodos, tanto para intentar disminuir la influencia de fenómenos ajenos al yacimiento como para el cálculo de la derivada de presión.

La evaluación de estos métodos se realizó cualitativa y cuantitativamente, teniendo en cuenta una metodología ya estudiada y comparando los resultados obtenidos con 
modelos teóricos que se fabricaron, soportados por herramientas software de simulación comerciales $e$ internacionalmente, respetados por la industria del petróleo, además, se trata un caso de campo para poder darle mucha más validez al estudio.

Uno de los grandes resultados del presente estudio es la herramienta Software "Derivar en Well", que permite ubicar la derivada de presión, no sólo, con los métodos tratados en este documento, sino también, con otros métodos bastante tratados en la literatura. Esta herramienta Software ha sido nutrida en la parte analítica, visual y de interfase por muchos integrantes del Grupo de Investigación de Pruebas de Presión (GIPP).

\section{Descripción del proyecto}

La idea principal de los algoritmos que se evaluaron, fue el ajuste de un arreglo de puntos "t vs. $\mathrm{P}$ " de una prueba de presión a una ecuación polinómica de grado $\mathrm{n}$, después de hallada la función es posible hallar la derivada de una manera sencilla.

Como las curvas de presión completas no se ajustan a ninguna curva polinómica, fue necesario evaluarla tomando grupos de puntos más pequerios, asi se toma un punto de trabajo y n puntos hacia delante y hacia atrás, al número de puntos auxiliares se les conoce como ventana, asi si se utilizan 4 puntos hacia delante y 3 hacia atrás la ventana es de 8 puntos.

Los algoritmos polinomiales que se avaluaron son:

- Minimos Cuadrados Móviles

- Savitzky-Golay

- Spline periódico

Para poder evaluar estos algoritmos se complementó la herramienta software: "Derivar en Well", la cual ha sido nutrida en la parte analítica, visual y de interfase por muchos integrantes del Grupo de Investigación de Pruebas de Presión (GIPP) de la Universidad Surcolombiana para luego poder comparar los resultados con modelos teóricos y así poder determinar los parámetros de los métodos más apropiados para la estimación de la función derivada de presión de las base de presión de pozo.

\section{Algoritmos}

La función derivada de presión, un concepto introducido por Tiab en 1976, ha llegado a ser la técnica más popular para analizar datos de pruebas de presión. La principal desventaja del uso de la función derivada es que esta construida por diferenciación numérica de datos discretos de presión. La derivada resultante es a menudo ruidosa y dificil de interpretar.

Los algoritmos polinomiales escogidos, Minimos Cuadrados Móviles, Savitzky-Golay, Spline periódico han devuelto muy buenos resultados en ingeniería de petróleos y en otras ciencias afines, por tal razón, se decidió evaluar estos algoritmos y así poder engrosar la caja de herramientas del ingeniero de petróleos encargado de la interpretación de pruebas de presión.

\section{Mínimos Cuadrados Móviles}

El objetivo de aplicar el método de mínimos cuadrados a una prueba de pozo es tratar de encontrar una función, en este caso polinómica que se ajuste a los pares de puntos $\mathrm{t}$ vs. $\mathrm{P}$, la forma generalizada de la ecuación que se busca es:

$y=\beta_{0}+\beta_{1} x+\ldots+\beta_{k} x^{k}$

En donde $\mathrm{K}$ sería el grado del polinomio deseado para nuestro ajuste.

Considerando la función:

$\mathrm{S}\left(\mathrm{b}_{0}, \mathrm{~b}_{1}, \ldots, \mathrm{b}_{\mathrm{k}}\right)$

Una condición necesaria para la existencia de un minimo relativo de esta función es que las derivadas parciales de $\mathrm{S}(\mathrm{b} 0, \mathrm{~b} 1, \ldots . ., \mathrm{bk})$ con respecto a bj, $\mathrm{j}=0,1,2, \ldots, \mathrm{k}$ sean cero. Resultando entonces las siguientes $\mathrm{m}+1$ ecuaciones lineales: 
g्gipp Evaluación de algoritmos para estimar la derivada de presión en pruebas de pozos

$$
\begin{aligned}
& b_{n} n+b_{1} \sum x_{i}+b_{2} \sum x_{1}^{2}+\ldots+b_{k} \sum x_{i}^{k}=\sum y_{i} \\
& b_{0} \sum x_{i}+b_{1} \sum x_{i}^{2}+b_{2} \sum x_{i}^{3}+\ldots+b_{k} \sum x_{i}^{k+1}=\sum x_{2} y_{i} \\
& \vdots \\
& \vdots \\
& b_{2} \sum x_{i}^{k}+b_{1} \sum x_{i}^{k+1}+\ldots+b_{k} \sum x_{i}^{2 k}=\sum x_{i}^{k} y_{i}
\end{aligned}
$$

Para resolver este sistema de ecuaciones se debe tener en cuenta que es extremadamente mal condicionada (ill-conditioned), esto significa que la familia de independencia lineal del polinomio $\left\{x^{k}\right]$ es muy cerrada para ser linealmente independiente. Esto quiere decir que todas las funciones $\mathrm{x}^{k}$ tienen una forma muy similar.

El sistema de ecuaciones, se intentó resolver por el método de LU decomposition, y el método de House Holder Transformation, obteniendo buenos resultados en unas pruebas, pero en otras simplemente no funcionaban. Por esta razón se decidió utilizar la subrutina DSOLV el cual resuelve el sistema satisfactoriamente.

Es bien sabido, que la curva de $\mathrm{t}$ us. $\Delta \mathrm{P}$ no tiene una tendencla polinómica clara, asi que a cada punto se le halla una función polinómica teniendo en cuenta cierta cantidad de puntos tanto adelante como detrás para luego hallar la derivada de la función hallada en cada tramo.

\section{Savitzky-Golay}

El método de mínimos cuadrados se puede simplificar si los puntos son equidistantes en el eje $\mathrm{X}$ ya que se puede evaluar un conjunto de datos equidistantes centrados en $\mathrm{X}=0$ (por que se sustrae $\overline{\mathrm{X}}$ ).

La matriz A del método de mínimos cuadrados móviles solo contiene términos en $\mathrm{X}$ y muchos de estos términos son 0 . Especialmente desde que el grupode datos es equidistante en $\mathrm{X}$, todas las sumas de los exponentes impares deben ser 0 . Por ejemplo:

$$
\begin{gathered}
\sum x=-2 \delta-\delta+0+\delta+2 \delta=0 \\
Y \\
\sum x^{3}=(-2 \delta)^{3}+(-\delta)^{3}+0+(\delta)^{3}+(2 \delta)^{3}=0
\end{gathered}
$$

Teniendo en cuenta esto Savitzky y Golay (1964) presentaron tablas de términos que permiten suavizar grupos de hasta 25 puntos (son utilizados solo puntos impares para poder reemplazar los puntos con ruido), en donde se pueden escoger los grados de los polinomios.

Considerando que las tablas presentadas por Savitzky-Golay, contenían un gran número de errores,Steinerycolaboradorespresentaronuna corrección en 1972 y después en 1978 Madden presentó formulas simples para calcular los convoluting integers $y$ asi poder implementarlos en un programa de computador.

Aunque el método de Mínimos Cuadrados en algunas ocasiones es considerado "Objetivo", aún quedan dos elecciones subjetivas para ajustar una curva a un polinomio móvil:

- El grado del polinomio

- La longitud de la ventana

En 1995 Barak extendió el método para permitir que el programa escogiera el grado del polinomio óptimo y asi el usuario sólo debe escoger el tamańo de la ventana y el grado de polinomio máximo. Esta auto-optimización funciona cada vez que los datos de la ventana cambian. La optimización está basada en una prueba $F$, usando un nivel de probabilidad predefinido del $5 \%$ y asi el programa puede escoger el grado de polinomio óptimo.

\section{Filtro Digital de Savítzky - Golay}

Siguiendo el mismo principio del método de Savitzky-Golay, para hallar la derivada de la curva de presión hay términos que sirven para reemplazar los datos de la ventana determinada por el usuario por una función polinómica y reduciendo el ruido que contiene la curva.

\section{Spline Periódico}

Si se desea construir una curva suavizada que pasa por un conjunto de datos dados $(\mathrm{x}, \mathrm{y})$ con un Spline cúbico y además se asume que los nodos están ordenados asi:

$$
a=x_{0}<x_{1}<x_{2}<\ldots<x_{n}=b
$$


Evaluación de algoritmos para estimar la derivada de presión en pruebas de pozos 㢦p

Entonces se puede representar la curva deseada por una función spline $\mathbf{S}$ con $S(x) \approx f(x)$, el cual está compuesta de polinomios cúbicos $\mathrm{Si}$ para $x \in\left[x_{i}+x_{i+1}\right]$ e $i=0, \ldots, n-1$.

Estos Si están definidos diferencialmente por cada subintervalo y puede satisfacer ciertas condiciones de conexión en los nodos.

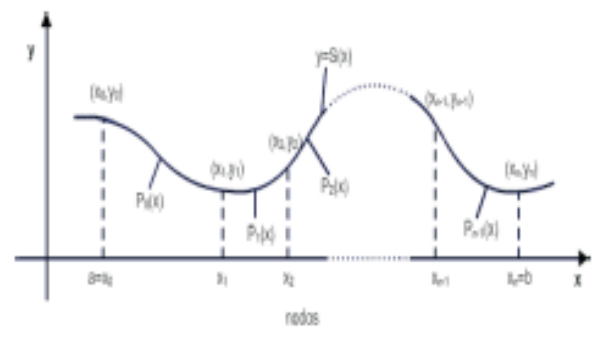

Figura 1. Spline Interpolation

Las condiciones de $\mathrm{X}_{0}$ y $\mathrm{X}_{\mathrm{n}}$ determinan el tipo de función Spline, en este caso para que sea periódico las condiciones son:

$$
\begin{array}{ll}
- & S\left(X_{0}\right)=S\left(X_{g}\right) \\
& (0.1) \\
& S^{\prime}\left(X_{o}\right)=S^{\prime}\left(X_{g^{\prime}}\right) \\
& (0.2) \\
& S^{\prime \prime}\left(X_{0}\right)=S^{\prime \prime}\left(X_{n}\right) \\
& (0.3)
\end{array}
$$

El algoritmo del método Spline Periódico es:

1. hacer $a_{i}=y i$ para $i=0, \ldots n-1$

2. hacer hacer $a_{0}=a, b_{0}=b_{n}, c_{0}=c_{n}$

3. resolver el sistema $\AA_{c}=\mathrm{g}$ con:

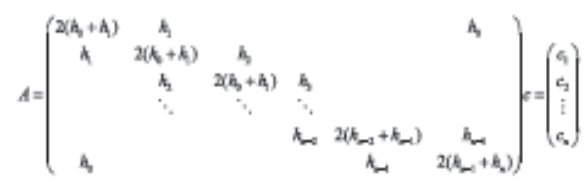

$$
\begin{aligned}
& g=\left(\begin{array}{c}
\frac{3}{h_{1}}\left(a_{2}-a_{1}\right)-\frac{3}{h_{0}}\left(a_{1}-a_{0}\right) \\
\frac{3}{h_{2}}\left(a_{3}-a_{2}\right)-\frac{3}{h_{1}}\left(a_{2}-a_{1}\right) \\
\vdots \\
\frac{3}{h_{r}}\left(a_{s+1}-a_{n}\right)-\frac{3}{h_{m}}\left(a_{s}-a_{n-1}\right)
\end{array}\right) \\
& \text { Con } \\
& a_{n+1}=a_{1}, a_{n}=a_{0}, c_{n}=c_{0}, h_{n}=h_{0}, \text { y } h_{i}=x_{i+1}-x_{i} \text { si } n \geq 3
\end{aligned}
$$

Para $\mathrm{n}=2$, la matriz A tiene la forma

$$
A=\left(\begin{array}{cc}
2\left(h_{0}+h_{1}\right) & h_{0}+h_{1} \\
h_{0}+h_{1} & 2\left(h_{0}+h_{1}\right)
\end{array}\right)
$$

4. hacer

$$
b i=\frac{1}{h_{i}}\left(a_{i+1}-a_{i}\right)-\frac{h_{i}}{3}\left(c_{i+1}-2 c_{i}\right) \text { para } i=0, \ldots,
$$

5. hacer $d i=\frac{1}{3 h_{1}}\left(c_{i+1}-c_{i}\right)$ para $i=0, \ldots, n-1$

Los resultados del análisis de los diferentes modelos se muestran en el siguiente orden.

- Tabla con las propiedades del yacimiento, pozo y fluido.

- Gráfico dt vs. dPy dP' del modelo

- Gráficos comparando los resultados de los métodos.

- En barras.

- En dispersión.

Tabla 1. Propiedades del Yacimiento y de los Fluidos -
Modelo 1

\begin{tabular}{|l|r|}
\hline \multicolumn{2}{|c|}{ Propiedades del Yacimiento $y$ de los } \\
Fluidos
\end{tabular}

Universidad Surcolombiana Facultad de Ingeniería 
दूgाpp Evaluación de algoritmos para estimar la derivada de presión en pruebas de pozos

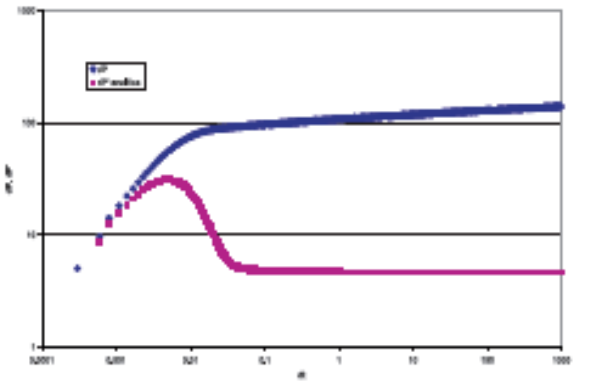

Figura 2. Modelo 1 - Vertical Homogéneo Infinito

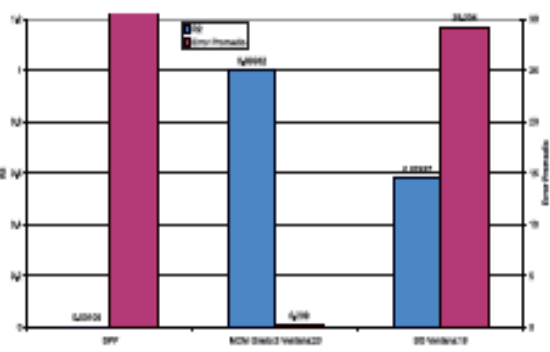

Pigura 3. Error Promedio y R2-Modelo 1

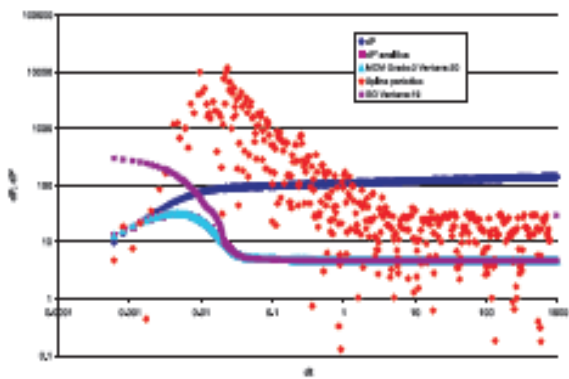

Figura 4. Comparación por dispersión - Modelo 1

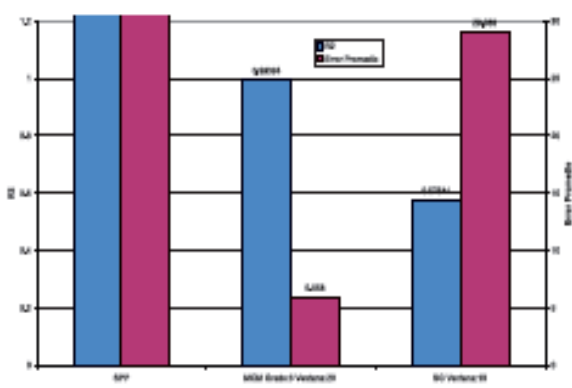

Figura 5. Error Promedio y R2-Modelo 1 (Ruido

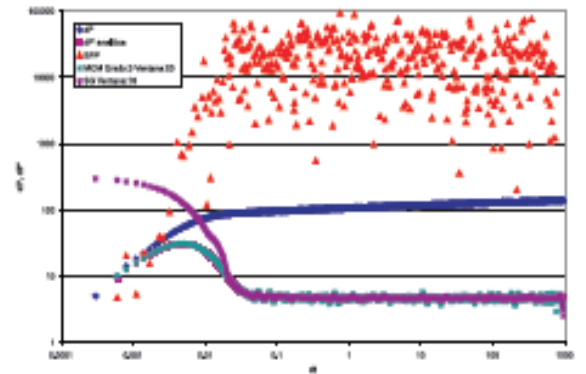

Pigura 6. Comparación por dispersión - Modelo 1 (Ruido)

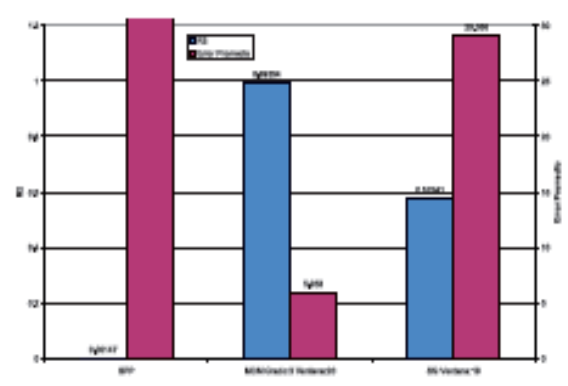

Figura 7. Error Promedio y R2-Modelo 1 (Suavizado con SMOOFT)

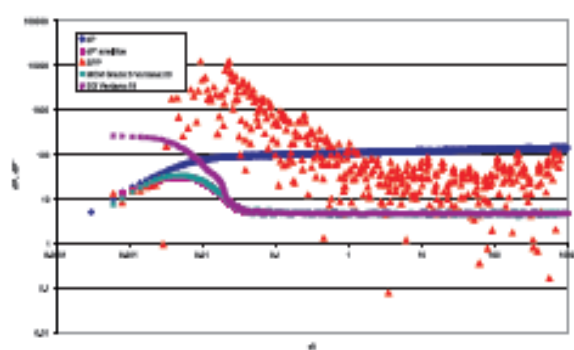

Pigura 8. Comparación por dispersion - Modelo 1 (Suavizado con SMOOFT)

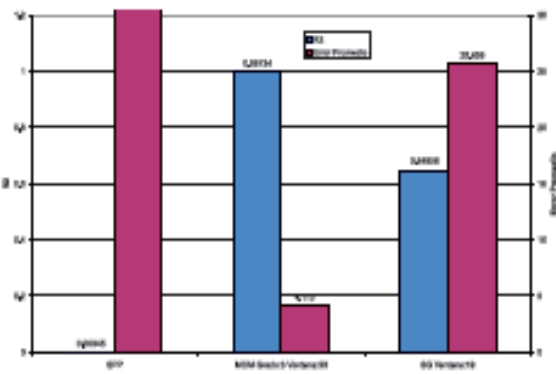

Plgura 9. Error Promedio y R2- Modelo 1 (Suavizado con Savitzky-Golay) 


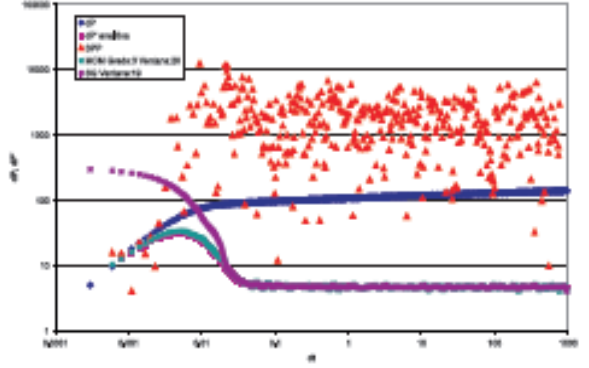

Figura 10. Comparación por dispersión - Modelo 1 (Suavizado con Savitzky-Golay)

Para los otros 6 modelos teóricos, se hizo el mismo proceso de comparación y asi fácilmente se puede determinar tanto cualitativa como cuantitativamente los algoritmos apropiados para estimar la derivada de presión de las pruebas de presión de pozo.

\section{》O Metodología}

Para evaluar estos algoritmos se aplicó una metodología probada no sólo en este trabajo, la cual brindó excelentes resultados. Utilizando Software comercial de simulación de yacimientos, se han crearon pruebas de presión teóricas con su respectiva derivada analítica para poder compararlos con los resultados de los métodos polinómicos. Los modelos de yacimiento escogidos son:

1. Vertical Homogéneo Infinito

2. Vertical Homogéneo Cerrado

3. Vertical Homogéneo Fallado

4. Vertical Naturalmente Fracturado

5. Vertical con Fractura de Conductividad Infinita

6. Vertical con Fractura de Conductividad Finita

Horizontal Anisotrópico y Horizontal Naturalmente

Fracturado.

Luego de tener los modelos listos se halló la derivada de presión con los algoritmos polinomiales variando los parámetros de los que dependen y los resultados se compararon con la derivada analítica teniendo en cuenta:
1. El error mínimo promedio:

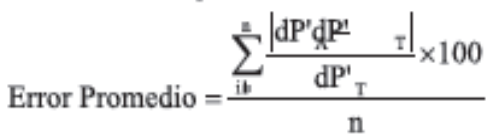

2. Coeficiente de determinación $\mathrm{R} 2$ que representa que tanto el modelo de regresión representa el modelo térico

$R^{2}=\frac{\left[n \sum\left(d P_{T}^{\prime} d P^{\prime}{ }_{A}\right)-\sum d P_{T}^{\prime} \sum d P_{A}\right]^{2}}{\left[n \sum d P_{T}^{2}-\left(\sum d P_{T}\right)^{2}\right]^{*}\left[n \sum d P^{\prime}{ }_{A}{ }^{2}-\left(\sum d P^{\prime}{ }_{A}\right)^{2}\right]}$

Donde:

n: número de datos.

dP'A: Derivada hallada con algoritmo.

dP'T: Derivada teórica.

Para estudiar la influencia del ruido en la determinación de la derivada de las pruebas de presión, se agregó ruido aleatoria a las pruebas anteriormente mencionadas y además de esto se sometió al siguiente proceso:

1. Hallar la derivada con la data ruidada y luego evaluar resultados

2. Hallar la derivada con la data suavizada con el método SMOOTF y luego evaluar resultados

3. Hallar la derivada con la data suavizada con el método de Savitzky-Golay y evaluar los resultados.

$\mathrm{Y}$ asi se determina con esta metodología si la derivada hallada con los métodos numéricos planteados funcionan para el procesamiento de las curvas de presión de pozo.

\section{bo Conclusiones}

- El algoritmo de Spline periódico, no es conveniente para hallar las derivadas de presión.

- Aunque se recomienda suavizar la derivada, en caso de que presente mucho ruido si ésta muestra mucho ruido. Las derivadas obtenidaspor el métododeSplinePeriódico. no mostraron resultados satisfactorios, aún después del suavizamiento. 
g्gाpp Evaluación de algoritmos para estimar la derivada de presión en pruebas de pozos

- Cuando la curva de presión no tiene ruido, para hallar la derivada es mejor utilizar el método de mínimos cuadrados móviles con un grado de polinomio pequeno (entre 3 y 4) y una ventana grande; mientras que cuando la curva está muy ruidada es mejor utilizar un grado de polinomio grande y una ventana mạ́s pequería.

- El método de Savitky - Golay aunque presenta un gran margen de error en la zona del almacenamiento de la derivada, en el resto de la curva muestra una tendencia bastante acertada yes apta para su posterior interpretación.

- El método que arrojó menos error promedio y mayor R2 es el de: Mínimos cuadrados Móviles.

- Aplicar un proceso de suavizamiento a la curva de presión antes de derivarla con el método de Savitky - Golay, arrojó mejores resultados que aplicar el proceso con el algoritmo SMOOFT.

- Para hacer el análisis de la desviación de las derivadas, no es suficiente con la interpretación del error promedio. El método puede arrojar grandes porcentajes de error y aún asi la derivada puede mostrar una tendencia, haciendo posible su interpretación.

\section{n@ Bíbliografía}

1. DE LEVIE, Robert. 2001. "How to Use Excels in Analytical Chemistry: And in General Scientific Data Analysis". Port Chester, NY, USA: Cambridge University Press

2. ESCOBAR, Freddy y MONTEALEGRE, Matilde. 2007 "Algoritmia y Métodos Numéricos con Aplicaciones Practicas en Ingenieria". Primera Edición, Editorial Universidad Surcolombiana. Neiva, $\mathrm{H}$, Colombia.

3. BELHADJ, Khaled. 2001. "Effective Tool for Smoothing Well Test Data". MSsc. Thesis. The University of Oklahoma..

4. ENGELN-MÜLLGES, Gisela \& UHLIG, Frank. 1996. "Numerical Algorithms with Fortran". Springer-Vrelag Berlin Heidelberg. Germany.

5. PRESS, William H., et al. 1996. "Numerical Recipes in Fortran 77 - The art of Scientific Computing". Second Edition, Cambridge University Press. Vol 1. Cambridge, USA.

6. http://mate.uprh.edu/ ${ }^{\sim} \mathrm{pnm} /$ notas4061/ leastsqr/leastsq.htm 